\title{
Silicose em ex-mineiros de extração de cobre
}

\section{Silicosis among former copper mine workers}

Fernanda de Freitas Luz $^{1}$

Volmar Carlos Stüker ${ }^{1}$

M arcelo Bradelli Trevisan ${ }^{1}$

Silvia Letícia M erceo Bacchi Cirino ${ }^{1}$

${ }^{1}$ Curso de Especialização em Farmacologiae

Toxicologia, Universidade

Luterana do Brasil. R. D.

Pedro II 1220 Cj. 316

Higienópolis. 90550-141

Porto Alegre RS.

hsmenezes@computech.com.br
Abstract There is a dearth of Brazilian studies analyzing the effect of ongoing exposure to silica among former copper mine workers, as radiographic readings for silicosis have received widespread medical attention. The scope of this study is to investigatethe clinical and radiological manifestations of silicosis among former copper mine workers. A sample of 100 former copper miners had their clinical and occupational histories re corded and underwent chest radiography in a cross-sectional study that included personal interviews in which their age, sex and time worked in copper extraction were recorded. Patients with recent chest X-Rays brought them with them, or they signed a term of consent and $X$-Rays were taken and analyzed by the radiologist using the Student t Test and Pearson test. Among the 100 miners, $35 \%$ had silicosis, $11 \%$ had conditions other than silicosis, such as tuberculosis, emphysema, chronic pulmonary obstructive disease, and $54 \%$ had no pulmonary lesions. The high incidence of silicosis reinforces the recommendation that exposure should be halted as soon as an Xray detects the condition.

Key words Silicosis, Copper, Chronic obstructive pulmonary disease
Resumo Existe escassez de estudos brasileiros analisando o efeito da exposição contínua à sílica em ex-trabalhadores de minas de cobre. O s achados radiográficos produzidos pela silicose têm recebido atenção médica especial. 0 objetivo deste estudo foi caracterizar clinica e radiologicamente a silicose em ex-mineiros de extração de cobre. Foi feito um estudo transversal com $100 \mathrm{ex}$-minei ros de extração de cobre. Os itens foram coletados através de entrevista pessoal, dados clínicos ocupacionais e foram realizados exames radiológicos. $\mathrm{Na}$ entrevista foi verificada a idade, o sexo e o tempo de trabalho na extração de cobre. Os pacientes que já realizaram exame radiológico de tórax trouxeram estes para comparação. Os mineiros assinaram um termo de consentimento para realização dos exames radiológicos o qual foi aplicado pelo radi ologista que os interpretou. Entre os 100 ex-mineiros, 35\% são portadores de silicose, $11 \%$ apresentaram alterações pulmonares que podem ter outras causas, como tuberculose, enfisema e doença broncopulmonar obstrutiva crônica, e 54\% não apresentaram nenhuma alteração pulmonar. A alta freqüência de silicose encontrada reforça a recomendação de suspender a exposição à sílica tão logo se tenha uma radiografia sugestiva da doença.

Palavras-chave Silicose, Cobre, Doença pulmonar obstrutiva crônica 


\section{Introdução}

A silicoseéum tipo depneumoconioseconhecida desde a Antigüidade, causada pela inalação de poeira conten do sílica livre cristalina ${ }^{1}$. É reconhecida na legislação brasileira como doença profissional ou do trabalho, abrangendo o conceito legal de acidente de trabalho. Milhares de novos casos são diagnosticados a cada ano em várias partes do mundo, predominando nos países em desenvolvimento ondeas atividades de exposição à sílica são freqüentes, sendo que em países desenvolvidos as pneumoconioses estão em significativo declínio. No Vietnam, é considerada a doença ocupacional mais prevalente, sendo uma das maiores causas de concessão de ben efícios previdenciários aos trabal hadores. $\mathrm{Na}$ China, durante o período de 1991-1995, foram documentados mais de 500 mil casos de silicose, na maior parte entre trabal hadoresidosos. $\mathrm{Na}$ Índia, ocorreuma prevalência de silicose de $55 \%$ entre os trabalhadores, na maioria deles jovens. No Brasil, o número de trabalhadores potencialmente expostos a poeiras contendo ślica é superior a 6 milhões, sendo cerca de 500 mil em mineração e garimpo. Antes da era industrial, a mineração e os trabaIhos artesanais eram capazes de produzir tal doença. Com a industrialização e a aceleração de processos geradores de poeiras houve um incremento das doenças relacionadas às poeiras minerais nos últimos 100 anos. 0 Brasil mantém concomitantemente as atividades extrativistas, no setor de mineração, com o crescimento industrial. 0 padrão de morbidade provavelmente acompanha o modelo econômico do país, com a presença de doenças pulmonares tanto no setor extrativista, o que nos paises desenvolvidos foi controlado com a finalização deste tipo de processo, quanto no setor industrial ${ }^{2}$.

A sílica é tóxica para o macrófago alveolar devido a suas propriedades de superfície que le vam à lise celular ${ }^{1}$. 0 desenvolvimento da doença apresenta um forte gradiente dose resposta em relação à exposição à sílica, inclusive com formas progressivamente mais graves. A pesar de conhecida essa relação dose resposta para a silicose, não existeum único índicequesintetizea exposição cumulativa, o que facilitaria a comparação dos trabal hadores expostos ${ }^{3}$.

No contexto geológico, onde está inserida a M ina do Camaquã, local de extração do cobre, a sílica está presente em abundância ${ }^{4}$. Esta mina surgiu e desenvolveu-se a partir de 1865, com a descoberta de jazidas de cobre, tendo o ouro e a prata como subprodutos. Atualmente, esgota- das as reservas de cobre, foram encerradas as atividades em abril de 1996. A silicose predispõe o organismo a uma série de co-morbidades, pulmonares e extrapulmonares, como a tuberculose, o enfisema, a limitação crônica ao fluxo aéreo, as doenças auto-imunes, a fibrose pulmonar e o câncer ${ }^{5}$.

A sílica, ou dióxido desilício, éum composto natural formado pelos dois elementos químicos mais abundantes na crosta terrestre, 0 oxigênio e o silício. É encontrada na natureza nas formas amorfae cristalina, quequando combinadas com metais e óxidos dão origem a silicatos como 0 talco, o feldspato, o caulim e a mica. A forma amorfa, embora não seja inerte, é menos tóxica do que a cristalina, sendo encontrada em rochas vulcânicas vitrificadas, em terras diatomáceas não aquecidas, na sílica gel, no vidro sintético e na lã de vidro ${ }^{6}$. A sílica cristalina que se encontra na areia e em diversas rochas, como 0 arenito, 0 granito e o síliex apresentam variado polimorfismo. A forma mais comum, eque corresponde à cerca de $12 \%$ da crosta terrestre, é 0 quartzo ${ }^{6}$.

A dispnéia de esforço éo sintoma que marca o quadro clínico da silicose, evolui lenta e progressivamente, terminando, nas formas graves, por incapacitar totalmente os pacientes para 0 trabalho. A fibrose pulmonar irreversível e freqüentemente progressiva, produzida pela silicose, leva ao aparecimento da doença pulmonar obstrutiva crônica e, nas fases finais do processo, à insuficiência cardíaca congestiva ${ }^{7}$.

Classicamente são descritas três formas clínicas distintas da silicose, a crônica, a acelerada e a aguda. A silicose crônica ocorreapóslongo tempo do início da exposição, o qual varia de 10 a 20 anos, e é caracterizada pela presença de pequenos nódulos difusos que predominam nos terços superiores dos pulmões ${ }^{1}$. Na silicose acelerada, ou subaguda, que ocorre normal mente após cinco a dez anos do início da exposição, encontram-se nódulos silicóticos, semelhantes aos da forma crônica, porém em estágios mais iniciais de desenvolvimento, com componente inflamatório intersticial intenso edescamação celular nos al véolos. A silicose aguda ocorre associada a exposições maciças de sílica livre, por períodos que variam de poucos meses até quatro ou cinco anos, e é representada pela proteinose alveolar associada a infiltrado inflamatório intersticial. ${ }^{8}$.

As manifestações clínicas de silicose podem ser uma broncopneumopatia crônica, insuficiência respiratória crônica, dispnéia, tosse freqüente e dolorosa, com expectoração mucopurulenta, hemoptóica ou pontilhada de partículas ou 
filamentosnegros, astenia intensa eemagrecimento. A conclusão prática é a da importância do diagnóstico precoce, principalmente por ser asilicose uma doença irreversível, sem tratamento e decujos fatores responsáveis por seu caráter evolutivo, pouco se conhece ${ }^{\text {. }}$.

0 diagnóstico da silicoseé baseado na radiografia de tórax, em conjunto com história clínica e ocupacional coerentes. Os exames clínico eradiológico podem não ser muito precisos no início da doença, pela falta de características dos sintomas iniciais e pelo fato da sílica ser transparente aos raios $X$. $O$ exame radiológico mostra as lesões provocadas pela sílica, e não sua presença no pulmão, sendo que $30 \%$ dos casos leves não aparecem nas radiografias 9 .

A progressão das lesões pode, além do aumento de profusão, mostrar um aumento no diâmetro médio dos nódulos, chegando à coalescência e a grandes opacidades. Estas últimas, normalmente, aparecem nos campos superiores e médios, crescendo em direção aos hilos e são usualmente bilaterais e simétricas. Com o tempo, essas massas ten dem a tracionar o parênquima, surgindo enfisema e bolhas no tecido adjacente. Essa condição também é conhecida como fibrose maciça progressiva ${ }^{10}$.

A importância do aparelho respiratório nas intoxicações émuito grande, pois além deconstituir via de absorção e excreção de tóxicos, écom grande freqüência prejudicado em suas funções pelos efeitos lesivos de substâncias tóxicas ${ }^{11}$.

A determinação de um limite de tolerância seguro para a sílica é difícil dada as múltiplas variáveis envolvidas, como a diferente patogenicidade dos diversos tipos de sílica, o efeito da exposi ção concomitante a outras poeiras, a variabilidade da resposta individual, etc ${ }^{12}$.

Uma vez reconhecida à presença de sílica respirável no ambiente de trabalho, medidas gerais, objetivando manter os teores de poeira em níveis aceitáveis, devem ser tomadas, como ventilação adequada eumidificação. N os locais em quea concentração persiste elevada, como nas situações de construção de túneis ou aplicação de jato de areia, a proteção efetiva ao trabalhador só poderá ser obtida isolando-o da poeira, suprindo-o dear proveniente de fonte exterior ao ambiente. Quando possível, a sílica deve ser substituída por outro material, como o pó de alumínio, atualmente empregado no polimento de peças de cerâmica ${ }^{12}$. De modo geral, as atividades demineração constituem uma das principais formas de exposição à sílica .

Osindivíduos expostosà sílica devem, periodicamente, ser submetidos a uma avaliação médica que inclua a radiografia do tórax. Detectada anormalidade compatível com a doença, a pessoa acometida deve ser afastada do contato com a poeira eorientada quanto aos direitos quea legislação Ihe faculta. Uma vez estabelecida a doença, não existe tratamento. $\mathrm{O}$ uso de Polivinil Piridina-N-óxido (PNO) foi experimentado, mas não há confirmação de sua efetividade em humanos ${ }^{12}$.

0 objetivo do presente estudo foi descrever freqüência de silicose e verificar os efeitos da exposição em ex-mineiros de extração de cobre.

\section{Casuística emétodos}

Foi realizado um estudo descritivo, transversal com ex-mineiros de extração de cobre das M inas do Camaquã, RS. Os ex-trabal hadores foram contatados após um levantamento dos registros de funcionários na Companhia Brasileira de Cobre (CBC). Sendo que destes, 100 ex-trabaIhadores foram pesquisados, considerando que os mesmos residiam no município.

As variáveis analisadas foram coletadas através de dadosclínicos deexamesocupacionais ede exames radiológicos. Foram coletadas informações através de entrevista pessoal devidamente validada pelo médico responsável, onde se constou a idade, o gênero e o tempo de trabalho na extração de cobre. 0 questionário aplicado na entrevista foi formulado com base em pesquisas realizadas anteriormente em casos de doenças de trabalho. Os mineiros pesquisados foram contatados especialmente para a realização da pesquisa. Cada paciente fez uma radiografia de tórax PA-P (posicionamento), solicitada por um médico de medicina do trabalho, cuja interpretação foi: trabalhadores portadores da doença e não portadores da doença. 0 diagnóstico radiológico foi baseado na Classificação Internacional de Radiografias de Pneumoconioses da OIT-2000.

Os exames foram realizados pelo Sistema Ú nico de Saúde. Os ex-trabalhadores foram avaliados durante o período de dois meses. Todos os pesquisados que já tivessem realizado exames de raios $X$ de tórax levaram estes para comparação.

As variáveis foram analisadas utilizando estatística descritiva, Teste t de Student e Correlação de Pearson. Foram analisados exames radiológicos, idade, gênero e tempo de exposição à sílica em 100 ex-mineiros deextração de cobre. 0 limite alfa considerado foi de $5 \%$, com nível de significância de 0,05.

Participantes da pesquisa assinaram um termo de consentimento para a realização dos exa- 
mes radiológicos, os quais foram aplicados pelo médico radiologista responsável pela interpretação das radiografias. 0 protocolo da pesquisa foi aprovado pelo Comitê de Ética em Pesquisa da Universidade Luterana do Brasil

\section{Resultados}

Dos 100 casos estudados verificou-se que 35\% dos trabalhadores eram portadores de silicose, sendo que estes tiveram tempo de exposição entre 4 e 40 anos (Tabela 1). Dos demais mineiros, $11 \%$ possuíam outras alterações pulmonares que poderiam ter sido provocadas por outros agentes como, tuberculose, enfisema e doença broncopulmonar obstrutiva crônica, estes pacientes ficaram expostos no mínimo 8 anos.

De acordo com os dados coletados, podemos afirmar que a causa de silicose nos mineiros deve-se ao fato de terem estado expostos à sílica durante suas jornadas de trabalhado. A comparação (Testet) das idades do grupo a com silicose e do grupo sem silicose $(p=0,09)$ e do tempo de exposição $(p=0,19)$ não revelou diferença estatística.

Dos 100 trabalhadores pesquisados, 35 apresentaram silicose $(\mathrm{N}=35)$ e tinham idade superior a 40 anos. A maior freqüência foi entre 60 e69 anos de idade (Tabela 2). A média da idade neste grupo foi 61 anos ( $D P=11,16)$ ea média de exposição foi de 15,7 anos ( $D P=5,7)$.

A maior freqüência de indivíduos sem silicose $(N=65)$ teve idade entre 50 e 59 anos (Tabela 2), média de 56,7 anos ( $D P=12,3)$, com tempo de exposição médio de 13,9 anos (DP=6,5).

Foi possível verificar que o maior número de trabalhadores que desenvolveram a doença são aqueles queestiveram expostos entre 10 e 19 anos (Tabela 1). Enquanto que o maior número de indivíduos que não desenvolveu a doença ficou exposto em tempo inferior a 10 anos.

Tabela 1. Percentual da doença deacordo com o tempo de exposição à sílica.

\begin{tabular}{lrrrr}
\hline & \multicolumn{2}{c}{$\begin{array}{c}\text { Com silicose } \\
\text { N }(\%)\end{array}$} & \multicolumn{2}{c}{$\begin{array}{c}\text { Sem silicose } \\
\text { N }(\%)\end{array}$} \\
\hline Tempo $0-9$ & 10 & $(28,6)$ & 29 & $(44,6)$ \\
Tempo $10-19$ & 21 & $(60,0)$ & 28 & $(43,1)$ \\
Tempo 20-29 & 3 & $(8,5)$ & 6 & $(9,2)$ \\
Tempo 30-39 & 1 & $(2,9)$ & 2 & $(3,1)$ \\
Total & 35 & $100 \%$ & 65 & $100 \%$ \\
\hline
\end{tabular}

No entanto, o percentual de indivíduos com a doença entre os 100 trabalhadores pesquisados foi de $35 \%$, índice considerado elevado.

Não houve correlação entre idade e tempo de exposição nem no grupo com silicose $(p=0,63)$ nem no grupo sem silicose $(p=0,40)$ (Teste Pearson).

\section{Discussão}

Em busca de silicose foram analisadas radiografias de 100 ex-mineiros de extração de cobre no estado do Rio Grande do Sul, Brasil, onde se verificou 35\% de indivíduos afetados, cifra superior a encontrada por Holanda et al. ${ }^{13}$ que encontraram uma prevalência de silicose de $27 \%$ em cavadores de poços, e inferior aos $42 \%$ encontrados por Carneiro ${ }^{5}$, embora este último tenha sido um estudo de pacientes hospitalizados. Podemos afirmar, de acordo com o estudo, que a prevalência de silicose em $21 \%$ dos mineiros, com exposição que varia de 10 a 15 anos é confirmada pela literatura ${ }^{10}$. De acordo com 0 estudo realizado por Castro et al. ${ }^{2}$, em levantamento nacional baseado em internações pelo SUS, a região Nordeste vivenciou no final dos anos 80 e início dos anos 90 um aumento de casos de silicoses relacionados à atividade de cavar poços, ondea ocorrência foi de $26,4 \%$ de silicóticos. Os autores verificaram também queo Estado de M inas Gerais possui o maior número de casos de internação por silicose do país. Segundo o M inistério da Saúde, até 1998 haviam sido diagnosticados mais de 7.416 casos de silicose na região de N ova Lima, área de mineração de ouro.

É conhecido que a silicose predispõe o organismo a uma série de outras doenças pulmonares, como a tuberculose, a fibrose pulmonar e 0 enfisema, por exemplo. Segundo Carneiro et al. ${ }^{3}$,

Tabela 2. Percentual da doença de acordo com a idade do ex-trabalhador.

\begin{tabular}{lrrrr}
\hline & \multicolumn{2}{c}{$\begin{array}{c}\text { Com silicose } \\
\mathrm{N}(\%)\end{array}$} & \multicolumn{2}{c}{$\begin{array}{c}\text { Sem silicose } \\
\mathrm{N}(\%)\end{array}$} \\
\hline Idade $30-39$ & 0 & $(0)$ & 5 & $(7,7)$ \\
Idade 40-49 & 7 & $(20,0)$ & 18 & $(27,7)$ \\
Idade 50-59 & 7 & $(20,0)$ & 20 & $(30,8)$ \\
Idade 60-69 & 11 & $(31,4)$ & 15 & $(23,1)$ \\
Idade 70-79 & 9 & $(25,7)$ & 2 & $(3,1)$ \\
Idade $80-89$ & 1 & $(2,9)$ & 4 & $(6,1)$ \\
Idade 90-99 & 0 & $(0)$ & 1 & $(1,5)$ \\
Total & 35 & $100 \%$ & 65 & $100 \%$ \\
\hline
\end{tabular}


que estudaram mineradores de ouro, a ocorrência de tuberculose é muito maior em silicóticos do que na população em geral. Foram encontrados no presente estudo dois indivíduos com silicose e portadores de tuberculose. Segundo a literatura, os dados que levam a essa suspeita são uma rápida progressão das lesões, formação de conglomerados e grandes opacidades, além dos sintomas constitucionais como astenia, emagrecimento efebrícula persistente ${ }^{1}$.

A exposição profissional à sílica no Brasil é mais alta do que na população européia, segundo pesquisa conduzida por Ribeiro et al. ${ }^{14}$, que estudaram a população brasileira utilizando dados do Relatório Anual de Informações Sociais. A ocorrência da silicose depende de vários fatores, dentreeles, a suscetibilidade individual, o tamanho das partículas, o tempo de exposição e a concentração de sílice livre respirável ${ }^{1}$. Podemos evidenciar na pesquisa realizada, que os pacientes portadores de silicose com 20 a 30 anos de exposição são em número menor do que aqueles que não possuem a doenças e que foram expostos duranteo mesmo período. A silicose, além de atingir os mineiros de cobre, como no presente estudo, também atinge trabalhadores da indústria cerâmica, fato quecomeçou a receber a devida importância com o estudo de N ogueira et al. ${ }^{15}$.

Por determinação legal do M inistério da Saúde e do M inistério do Trabalho, os trabalhadores devem ser radiografados anualmente e submetidos a testes espirométricos, a cada dois anos, como forma de detectar precocemente as altera- ções pulmonares². O Programa Nacional de Eliminação da Silicose traçou como meta a diminuir a incidência de silicose até 2015 e eliminar a silicose como problema desaúdepública no Brasil até $2030^{16}$. É provável que fiscalização nos limites de exposição à sílica, estabelecidos desde 1978 pelo M inistério do Trabalho ${ }^{16}$, nos trabalhadores da mina aqui estudada, tenha sido deficientee colaborado com o alto índice de silicose encontrado. Em recente estudo, $M$ endonça et al. ${ }^{17}$ relataram a relação do tempo de exposição a fatores de risco, como a areia, em trabalhadores de fundição. Esta relação com o tempo de exposição também ocorreu no presente estudo, e embora a população estudada tenha sido de mineiros de cobre 0 padrão de tempo de exposição segue a mesma tendência.

Verificou-sequenaliteratura científica brasileira atual sobre pneumoconiose, os estudos epidemiológicos sobre silicose são relativamente escassos.

\section{Conclusão}

A alta prevalência de silicose encontrada reforça a recomendação de afastar os trabalhadores da exposição à sílica tão logo se tenha uma radiografia sugestiva da doença.

Os mineiros expostos à sílica apresentaram doenças pulmonares, além de silicose, que podem estar relacionadas à vulnerabilidade que a atividade profissional destes pode propiciar.

\section{Colaboradores}

FF Luz realizou a coleta de dados e digitação do texto; VC Stüker realizou e interpretou os exames; M B Trevisan e SLM B Cirino fizeram análise e formatação final do texto; HS M enezes orientou todas as fases do estudo e fez a revisão bibliográfica final do artigo. 


\section{Referências}

1. M endes R. Patologia do trabalho atualizada e ampliada. São Paulo: Atheneu; 2003.

2. Castro HA, Silva CG, Vicentin G. Estudo das internações hospitalares por pneumoconiose no Brasil, 1984-2003. Rev Bras Epid 2005; 8(2):150-160.

3. Carneiro APS, Barreto SM, Siqueira AL, Rocca PF. Índice de exposição à sílica na atividade de mineração de ouro. Rev Saude Publica 2006; 40(1):83-91.

4. Teixeira G, Gonzales AP, organizadores. Principais depósitos minerais do Brasil. Brasília: DNPM; 1988.

5. Carneiro APS, Campos LO, Gomes M FCF, Assunção AA. Perfil de 300 trabalhadores expostos à sílica atendidos ambulatorialmente em Belo Horizonte. J Pneumol 2002; 28(6):1-6.

6. Terra Filho M, Santos UP. Silicose. J Bras Pneumol 2006; 32(Supl. 2):41-47.

7. M endes R. M edicina do trabalho e doenças profissionais. São Paulo: Sarvier; 1980.

8. Fraser RG, Peré JAP. Diagnosis of diseases of the chest. Barcelona: Salvat; 1979.

9. Bellusci SM. Doenças profissionais ou do trabalho. São Paulo: Senac; 1996.

10. Paul LW, Juhl JH. Interpretações radiológicas. 3a ed. Rio de Janeiro: Sarvier; 1977.

11. Schvartsman S. Intoxicações agudas. São Paulo: Sarvier; 1979.

12. Silva LCC. Compêndio de pneumologia. $2^{2}$ ed. São Paulo: BYK; 1991.

13. Holanda M A, Holanda MA, Martins MP, Felismino PH, Pinheiro VG. Silicosis in Brazilian pit diggers: relationship between dust exposure and radiologic findings. Am J Ind M ed 1995; 27(3):367-378.

14. Ribeiro FSN, Camargo EA, Algranti E, Wünsch FiIho V. Exposição ocupacional à sílica no Brasil no ano de 2001. Rev bras epidemiol 2008; 11(1):89-96.

15. Nogueira DP, Certain D, Brólio R, Garrafa NM, Shibata H. Ocorrência de silicose entre trabalhadores da indústria cerâmica da cidade de Jundiaí, SP(Brasil). Rev Saude Publica 1981; 15(3):263-271.

16. Algranti E, Handar Z, Ribeiro FSN, Bon AMT, Santoa $A M$, Bedrikow B. Exposição à sílica silicose e 0 Programa Nacional de Eliminação da Silicose. Ciencia $Y$ trabajo 2004; 6(1):1-13.

17. M endonça $\mathrm{EM}$, Silva RC, Bussacos MA, Algranti $\mathrm{E}$. Respiratory impairment in Brazilian foundry workers exposed to sand. Am J Ind M ed 2007; 50(2): 83-91.

Artigo apresentado em 09/03/2008

Aprovado em 23/06/2008

Versão final apresentada em 15/07/2008 